\title{
Gonadal Steroids Promote Glial Differentiation and Alter Neuronal Morphology in the Developing Hypothalamus in a Regionally Specific Manner
}

\author{
Jessica A. Mong,, ${ }^{1}$ Edmund Glaser, ${ }^{2}$ and Margaret M. McCarthy ${ }^{2}$ \\ ${ }^{1}$ Department of Pharmacology and Experimental Therapeutics and ${ }^{2}$ Department of Physiology and Program in \\ Neuroscience, University of Maryland School of Medicine, Baltimore, Maryland 21201
}

One of the more striking sexual dimorphisms in the adult brain is the synaptic patterning in some hypothalamic nuclei. In the arcuate nucleus (ARC) males have twice the number of axosomatic and one-half the number of axodendritic spine synapses as females. The opposite pattern is observed in the immediately adjacent ventromedial nucleus (VMN). In both cases, early exposure to testosterone dictates adult dimorphism, but the exact timing, mechanism, and site of steroid action remain unknown. Astrocytes also exhibit sexual dimorphisms, and their role in mediating neuronal morphology is becoming increasingly evident. Using Golgi-Cox impregnation to examine neuronal morphology and glial fibrillary acidic protein immunoreactivity (GFAP-IR) to characterize astrocytic morphology, we compared structural differences in dendrites and astrocytes from the $A R C$ and $V M N$ in postnatal day 2 rat pups from four hormonally different groups. Consistent with previous observations, testosterone exposure induced a rapid and dramatic stellation response in ARC astrocytes. Coincident with this change in astrocytic morphology was a $37 \%$ reduction in the density of dendritic spines on ARC neurons. In contrast, astrocytes in the VMN were poorly differentiated and did not respond to testosterone exposure, nor were there any changes in neuronal dendrite spine density. However, VMN neurons exposed to testosterone had almost double the number of branches compared with that in controls. These data suggest that the degree of maturation and the differentiation of hypothalamic astrocytes in vivo are correlated with the ability of neurons to sprout branches or spines in response to steroid hormones and may underlie regionally specific differences in synaptic patterning.

Key words: sexual differentiation; arcuate; ventromedial nucleus; estrogen; spine density; developmental synaptic plasticity; GFAP; Golgi-Cox
The perinatal developmental period is characterized by dramatic sex differences in circulating gonadal steroids in a variety of mammalian species including humans (Gerall et al., 1992). On embryonic day 18, the testes of developing male rats secrete adult levels of testosterone that gradually decline until a second peak of secretion on the day of birth. In females, exposure to gonadal steroids remains uniform and low (Weisz and Ward, 1980). This dimorphic exposure to steroids has profound and permanent effects on the developing brain. In the rodent, it is well established that estrogens, the products of aromatizable androgens, mediate many aspects of sexual differentiation of the male brain during a restricted development period from embryonic day 18 to postnatal day 6-10 (Maclusky and Naftolin, 1981; Arnold and Gorski, 1984; Gerall et al., 1992). Exposure to estrogens during this critical period results in a "masculinized" neural pattern in the adult rat, whereas a lack of such exposure yields a "feminized" brain. A major consequence of this dissimilar steroid exposure is the sexually dimorphic synaptic patterning in the arcuate nucleus (ARC) and ventromedial nucleus (VMN), two hypothalamic nuclei that play critical roles in the control of gonadotropin

\footnotetext{
Received Aug. 6, 1998; revised Oct. 29, 1998; accepted Dec. 2, 1998.

This work was supported by National Science Foundation Grant IBN-9511328 to M.M.M. We would like to thank Drs. Greg Ball, Anne Murphy, and Scott Thompson for helpful comments on this manuscript.

Correspondence should be addressed to Jessica A. Mong, Department of Pharmacology, University of Maryland, 655 West Baltimore Street, Baltimore, MD 21201.

Copyright (C) 1999 Society for Neuroscience $\quad 0270-6474 / 99 / 191464-09 \$ 05.00 / 0$
}

secretions from the pituitary (Ojeda and Urbanski, 1994) and stereotypic female sex behavior (Pfaff et al., 1994), respectively. Quantitative electron microscopy studies have shown that in the ARC, females have approximately twice the number of axodendritic spine synapses as males and this patterning is sensitive to hormonal manipulation during the critical period for brain sexual differentiation (Matsumoto and Arai, 1980). The opposite pattern is observed in the ventrolateral VMN where adult males have twice as many dendritic spine synapses as females (Matsumoto and Arai, 1986).

The cellular and molecular mechanisms mediating steroidinduced changes in synaptic patterning are unknown. Recently, several lines of evidence have focused on neuronal-glial interaction as a possible mechanism for mediating synaptic connectivity. We have demonstrated previously that astrocytes in the ARC of neonatal rats exhibit a sexually dimorphic morphology as early as postnatal day 1 (PN1). In gonadally intact males, the ARC has a greater frequency of fully differentiated astrocytes, as evidenced by their complex morphology. As with synaptic patterning, this sex difference in astrocyte morphology is sensitive to hormonal manipulation. Castrate males and females treated with testosterone propionate demonstrated dramatic increases in astrocyte differentiation, whereas removal of testosterone by gonadectomy resulted in a lack of differentiation within $24 \mathrm{hr}$ (Mong et al., 1996). These data suggest that astrocytes may participate in synaptic patterning by guiding or blocking the path of neurites. As a first step in testing the hypothesis that steroid-mediated changes in astrocytic morphology determine sexually dimorphic 
A
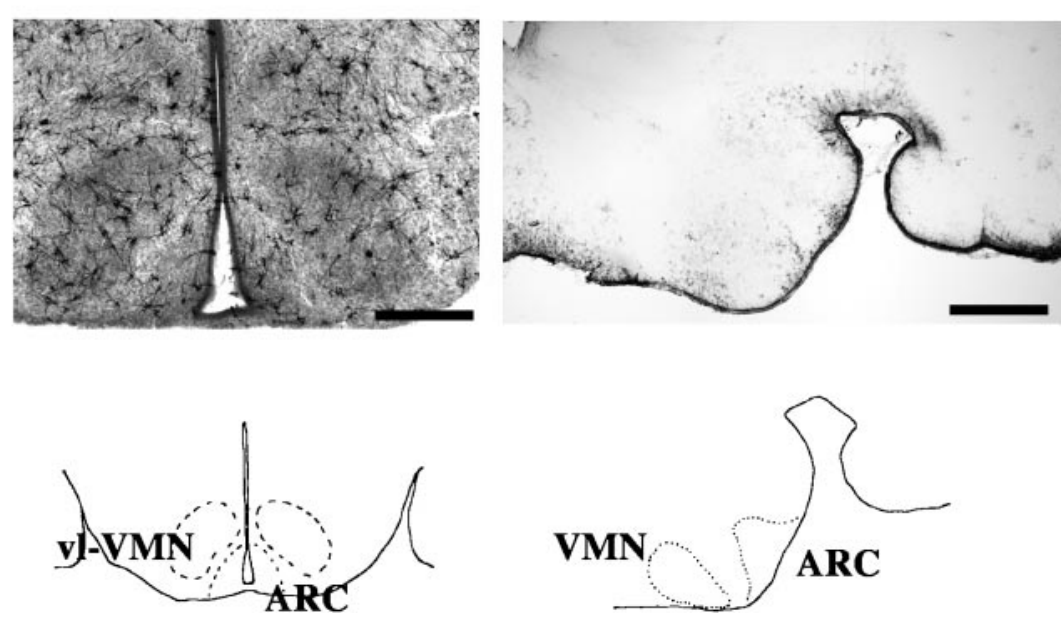

Figure 1. Anatomical regions analyzed for neuronal and astrocytic morphology. $A$, Representative photomicrographs $(2 \times)$ of a coronal section from a Golgi-Coximpregnated PN2 rat brain (top) and camera lucida drawings of the $A R C$ and $V M N$ in the coronal plane (bottom). Impregnated neurons were reconstructed with computerized microscopy from the ventrolateral portion of the $V M N(v l-V M N)$ and the $A R C . B$, Representative photomicrographs $(2 \times)$ of GFAP-IR in the sagittal plane of a PN2 rat (top) and camera lucida drawings of the $A R C$ and $V M N$ in the sagittal plane of a neonatal rat brain (bottom). The astrocyte process length and the degree of differentiation were analyzed in the $A R C$ of sagittal sections to avoid the appearance of GFAP-IR tanicytes that are specialized glia present in the $A R C$ and median eminence. Scale bar, $500 \mu \mathrm{m}$. synaptic patterning, we have observed coordinated changes in the dendritic morphology of Golgi-Cox-impregnated neurons and in the morphology of astrocytes expressing glial fibrillary acidic protein (GFAP) in the ARC and VMN of PN2 rats that had been hormonally manipulated on the day of birth and on PN1. We examined regionally specific astrocyte maturation further by Western blot analysis of GFAP levels.

\section{MATERIALS AND METHODS}

\section{Animals}

Female Sprague Dawley rats (Charles River Laboratories, Wilmington, MA) were mated in our animal facility, and pregnancy was confirmed by the presence of sperm in the vaginal smear. Pregnant females were isolated and allowed to deliver normally. Animals were maintained on a reversed 12:12 hr light/dark cycle (lights out at 10:00 A.M.) and were given food and water ad libitum. Cages were checked regularly for the presence of pups to determine the day of birth (PN0). Only litters that were found in the morning were used in subsequent experiments. Castrations were performed on PN0 under cold anesthesia, and gonadally intact male and female pups underwent sham operations. All pups were toe-clipped for group identification. After being warmed to $37^{\circ} \mathrm{C}$, pups were returned to their mothers. All the pups received two subcutaneous injections $24 \mathrm{hr}$ apart of either $100 \mu \mathrm{g}$ of testosterone propionate (TP) in a $0.1 \mathrm{cc}$ volume of sesame oil or $0.1 \mathrm{cc}$ of sesame oil alone. Brains were collected on PN2, $\sim 24 \mathrm{hr}$ after the last hormonal treatment. The treatment groups were as follows: (1) castrate males injected with oil vehicle $(n=6) ;(2)$ gonadally intact males injected with oil vehicle $(n=8) ;(3)$ gonadally intact females injected with TP $(n=6)$; and (4) gonadally intact females injected with oil vehicle $(n=7)$.

\section{Golgi-Cox impregnation}

Brains were embedded according to the methods of Glaser and Van der Loos (1981) with some modifications. Briefly, the neonates were overdosed with pentobarbital before being transcardially perfused with $0.9 \%$ saline, and the brains were placed in $30 \mathrm{ml}$ of Golgi-Cox solution (1:1 solution of $5 \% \mathrm{~K}_{2} \mathrm{Cr}_{2} \mathrm{O}_{7}$ and $5 \% \mathrm{HgCl}_{2}$, which is then added to $5 \%$ $\mathrm{K}_{2} \mathrm{CrO}_{4}$ in a 4:10 ratio). The brains were placed in fresh Golgi-Cox solution after $48 \mathrm{hr}$ and remained in it for $20 \mathrm{~d}$. After impregnation, the brains were placed in $30 \%$ sucrose in $\mathrm{dH}_{2} \mathrm{O}$ for $3 \mathrm{~d}$, then cut on a vibratome at $100 \mu \mathrm{m}$, and mounted on $2 \%$ gelatin-subbed glass slides as described previously (Forgie et al., 1996; Gibb and Kolb, 1998). The sections were developed and counterstained with methylene blue according to the procedure outlined in Glaser and Van der Loos (1981).

\section{Immunocytochemistry: GFAP immunoreactivity}

Animals used for GFAP immunoreactivity (GFAP-IR) received the same hormonal treatment ( $n=4$ for each group) as the Golgi-Coximpregnated brains and were killed by decapitation $\sim 24 \mathrm{hr}$ after the last hormonal treatment (PN2). The brains were removed and submersion fixed in $0.1 \%$ acrolein and $4 \%$ paraformaldehyde, followed by incubation in $30 \%$ sucrose in PBS for cyroprotection. After fixation, the brains were frozen on dry ice and stored at $-80^{\circ} \mathrm{C}$ until processed for immunocytochemistry.

Brains were sectioned into $50-\mu \mathrm{m}$-thick sections along the sagittal plane using a cryostat. The sections were placed into wells containing 0.1 $M$ PBS, washed twice in PBS, and incubated with $1 \% \mathrm{H}_{2} \mathrm{O}_{2}$ in $100 \%$ methanol for $15 \mathrm{~min}$. They were then washed three times in PBS, followed by a $1 \mathrm{hr}$ incubation in 10\% normal goat serum and $0.3 \%$ Triton $\mathrm{X}-100$ in PBS. After blocking, a mouse monoclonal GFAP primary antibody (Boehringer Mannheim, Mannheim, Germany) was applied to the sections at a 1:10,000 dilution in 10\% normal goat serum and $0.3 \%$ Triton X-100 in PBS, and the sections were incubated overnight at $4^{\circ} \mathrm{C}$. After primary incubation, the sections were washed three times in PBS and incubated in biotinylated secondary antibody (goat anti-mouse; Vector Laboratories, Burlingame, CA) for $1 \mathrm{hr}$, followed by three washes in PBS. Finally, the samples were incubated with an avidin-biotin horseradish-peroxidase complex (Vectastain ABC, Elite Kit; Vector Laboratories) for $1 \mathrm{hr}$ at room temperature, washed twice with PBS, and visualized with $0.05 \%$ 3,3'-diaminobenzidine tetrahydrochloride (Polysciences, Warrington, PA) and $0.005 \% \mathrm{H}_{2} \mathrm{O}_{2}$. After visualization, the sections were mounted serially on $2 \%$ gelatin-coated glass slides.

\section{Morphological analysis}

Dendrites. Golgi-Cox-impregnated dendrites were analyzed by computerized reconstruction (Glaser and Van der Loos, 1981). Slides were numerically coded, and the reader was blind to the experimental group. Anatomically matched sections (two to three) were analyzed from each animal for all groups. These sections corresponded to embryonic day 22 (E22) coronal plate 15 in the prenatal rat atlas of Altman and Bayer (1995) and contained both the ventrolateral VMN and a rostral portion of the ARC (Fig. 1A). Neurons that appeared well impregnated were marked for analysis. A $20 \times$ Nikon objective was used for this selection procedure so that the observer would not be biased by the appearance of spines on the dendrites. A neuron was considered well impregnated if its cell body was filled with the black precipitate and the dendrites were filled to their ends. Because axons were only occasionally filled, and rarely to their ends, their impregnation was not a criterion for determining well-impregnated neurons. Ten to twenty dendrites in both the VMN and ARC of each animal were randomly selected and analyzed for all groups. Well-impregnated dendrites were then reconstructed through the different focal planes of the section under a $100 \times$ oil-immersion objective using Neurolucida, an image-combining computer microscopy program (MicroBrightField, Colchester, VT). In the dendritic reconstruction, spines, branch points, and total dendritic length were measured. A spine was defined as any protrusion under $5 \mu \mathrm{m}$, and any protrusion over $5 \mu \mathrm{m}$ in length was considered to be a branch point. Branch points represented the number of times a primary dendrite divided into subsequent branches. Total dendritic length was measured in micrometers and includes all branches on the primary dendrite. Spine density was calculated by dividing the total number of spines per dendrite by the total length of 
the dendrite. Spine densities and total dendritic length were reported as the means for each treatment group.

GFAP-IR astrocytes. In sagittal sections, the arcuate nucleus appears as a distinct cell population in the region just lateral to the third ventricle. It was necessary to section the tissue for GFAP-IR in the sagittal plane because in coronal sections differentiated GFAP-IR astrocytes are obscured by GFAP-IR tanicytes, specialized glial cells found in the ARC and median eminence. The VMN also appears in the same sections as the ARC. It is an oblong-shaped nucleus just anterior to the ARC (Fig. $1 B)$. To analyze cells from the same anatomical region of the ARC and VMN for each animal, we selected the first four sections starting $\sim 100$ $\mu \mathrm{m}$ lateral to the third ventricle. These sections corresponded to E22 sagittal plate 2 in the prenatal atlas of Altman and Bayer (1995) (Fig. $1 B)$. Slides were numerically coded, and the investigator conducting the analysis was blind to the experimental group. GFAP-IR cells that could be identified as one individual cell were selected for analysis. The National Institutes of Health IMAGE software program was used to measure the process length of GFAP-immunoreactive cells in the ARC of anatomically matched sections across the four groups. The process length of GFAP-IR cells in the VMN was not quantifiable (see Results). By the use of the line selection tool, the path of a GFAP-IR process was traced from the center of the cell soma to its end. Using the measurement option, we measured the process length in micrometers. Three to five processes were measured per cell, and their mean process length per cell was determined. Seven to ten cells per section were selected for measurement, and four sections per brain from four animals per group were analyzed.

For further analysis, all the GFAP-IR glial cells measured for process length in the ARC of the four treatment groups were then categorized into four arbitrary classes based on their morphological shape and degree of branching. This classification scheme was designed after observing distinct differences in glia morphology between the treatment groups (Mong et al., 1996). Class I cells were characterized as having only primary processes that were short and thick. Class II and class III cells were more bipolar in shape with extended processes. Class II cells possessed primary and some secondary processes for a total of 5-15, whereas class III cells had more secondary processes, usually 15-30. Finally, class IV cells were characterized by their long thin extended processes that possessed a high degree of branching (secondary and tertiary processes). In addition, the class IV cells were stellate in contrast to the bipolar morphology of classes I-III. Representative photomicrographs of the GFAP-IR astrocytes from the different classes are presented in Figure 2. Only GFAP-IR cells that were distinguishable as individual astrocytes were classified. Approximately 25 GFAP-IR cells per animal and four animals per group were classified. After classification, the percentage of astrocytes in each class was determined from the total number of cells classified for that brain.

\section{Western blotting}

Unmanipulated male and female pups $(n=7)$ were killed by decapitation $\sim 48 \mathrm{hr}$ after birth (PN2). The brains were removed and frozen on dry ice and stored at $-70^{\circ} \mathrm{C}$ until being processed for micropunching. Brains were cut in the coronal plane into $300-\mu \mathrm{m}$-thick sections on a cryostat. The sections were collected onto glass microscope slides and immediately frozen. The ARC and ventrolateral VMN were microdissected according to the micropunch technique of Palkovits (Palkovits and Brownstein, 1988). The tissue punches were placed in $50 \mu$ l of 10 mM HEPES, pH 7.2, and sonicated for $10 \mathrm{sec}$. An aliquot was removed to determine protein content, and the remaining tissue was denatured with SDS (1\% final concentration; Sigma, St. Louis, MO) and $\beta$-mercaptoethanol (1\% final concentration; Sigma) and stored at $-70^{\circ} \mathrm{C}$ until processing (Laemmli, 1970; Towbin et al., 1979)

Protein $(5 \mu \mathrm{g})$ from the $\mathrm{ARC}$ and $\mathrm{VMN}$ was electrophoresed in separate lanes on an $8-16 \%$ precast SDS-polyacrylamide gel (Novex, San Diego, CA) and transferred to a polyvinyl difluoride membrane (BioRad, Hercules, CA). Membranes were incubated for $3 \mathrm{hr}$ at room temperature with a 1:20,000 dilution of a mouse monoclonal antibody against GFAP (Boehringer Mannheim), followed by a 30 min incubation with a 1:3000 dilution of horseradish peroxidase-conjugated anti-mouse IgG (New England Biolabs, Beverly, MA). The Phototope chemiluminescence system (New England Biolabs) was used for detection of the protein recognized by the antisera. The blots were exposed to Hyperfilm-ECL (Amersham, Arlington Heights, IL) for varying exposure times. The protein was detected as a band of relative molecular mass of $51 \mathrm{kDa}$. After blot visualization, the membranes were stripped with
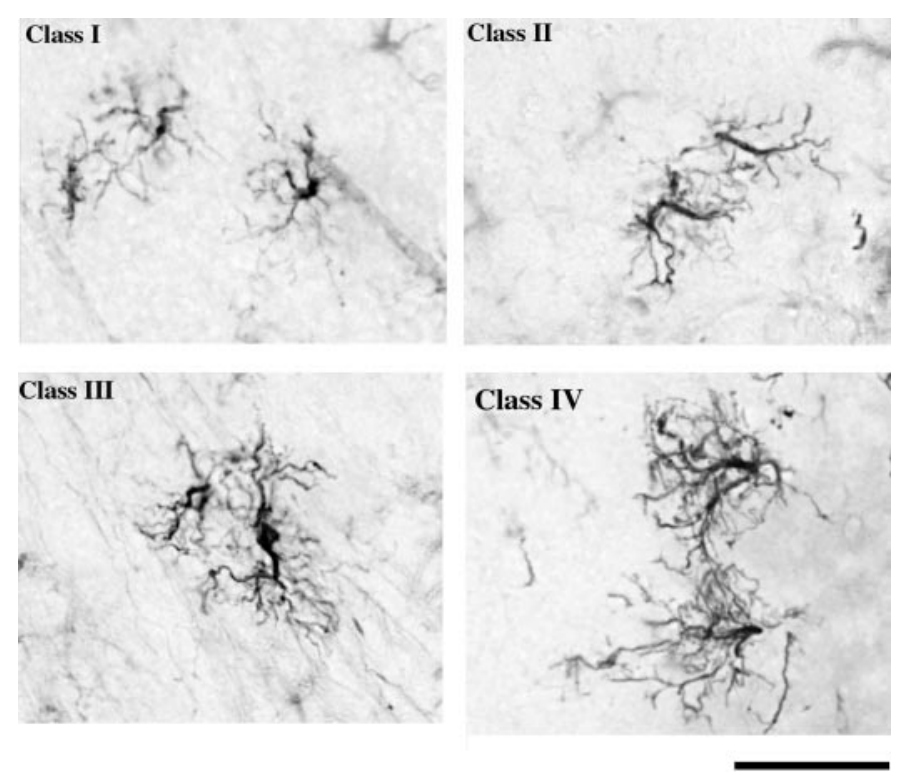

Figure 2. Representative photomicrographs $(40 \times)$ of classes of GFAP-IR cells in the ARC of PN2 rats. To characterize hormonemediated changes in astrocyte morphology further, we developed a classification scheme that divided cells into four arbitrary classes based on increasing complexity. Class I cells are characterized by only primary processes that extend from the soma. Class II cells have an overall bipolar shape, and their processes branch $\sim 5-15$ times. Class III cells are characterized by a more stellate appearance but still retain some bipolar morphology and have an increased number of branches (15-30) compared with that in Class II. Class IV cells are characterized by a fully stellate morphology. Scale bar, $25 \mu \mathrm{m}$.

ImmunoPure IgG Elution Buffer (Pierce, Rockford, IL) for $3 \mathrm{hr}$ at room temperature and then blotted with a 1:20,000 dilution of the antibody to the housekeeping gene glyceraldehyde-3-phosphate dehydrogenase (GAPDH) (Chemicon, Temecula, CA) as a control. The blots were visualized as described above. The immunoreactive band densities were quantified with a CCD camera and densitometry using National Institutes of Health IMAGE software. The GFAP-immunoreactive bands were standardized to the bands immunoreactive for GAPDH (relative molecular mass of $36 \mathrm{kDa}$ ), and the data are expressed as a ratio of GFAP optical density to GAPDH optical density.

\section{Statistical analysis}

Measurements for the ARC and VMN were done in the same brain and are therefore not entirely independent of each other. However, an ANOVA that treated each brain area as a repeated measure would fail to detect the significant effects of hormone treatment that occur in only one of the two areas. Thus, the comparison of the number of branches on primary dendrites and the spine density between the four treatment groups was analyzed by a two-way ANOVA with region and treatment as factors. All ANOVAs were followed by a Newman-Keuls post hoc test to determine significance between the groups. The regional comparison of dendritic length from the ARC and VMN as well as GFAP-IR process length in the ARC from the four treatment groups was analyzed by a one-way ANOVA. The data on the frequency distribution of the different classes were analyzed by chi-square $\left(\chi^{2}\right)$. GFAP expression in the ARC and VMN assayed by Western blotting was analyzed by Student's $t$ test. All statistical tests were conducted using the GB-STAT program on a Macintosh computer.

\section{RESULTS}

\section{Neuronal morphology}

Incubation of PN2 brains in Golgi-Cox solution for $20 \mathrm{~d}$ yielded 15-20 well-impregnated neurons per slice in the ARC and 20-25 well-impregnated neurons per slice in the VMN. Neurons in the ARC appeared as bipolar cells with approximately two to 
A

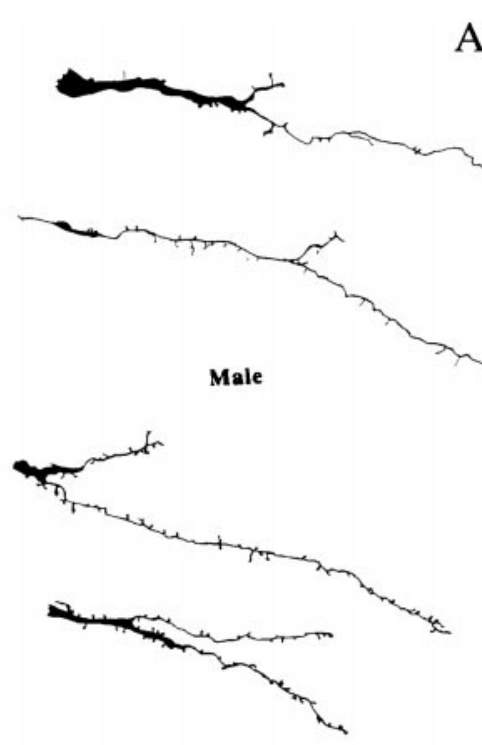

Castrate Male
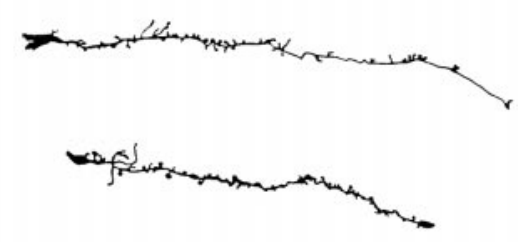

Female

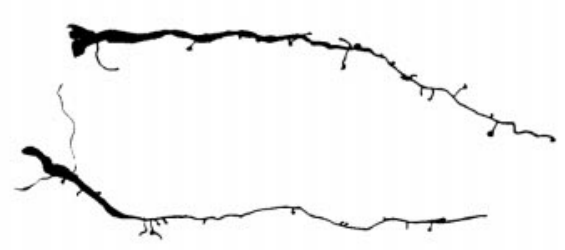

Female + TP

B

\section{VMN}
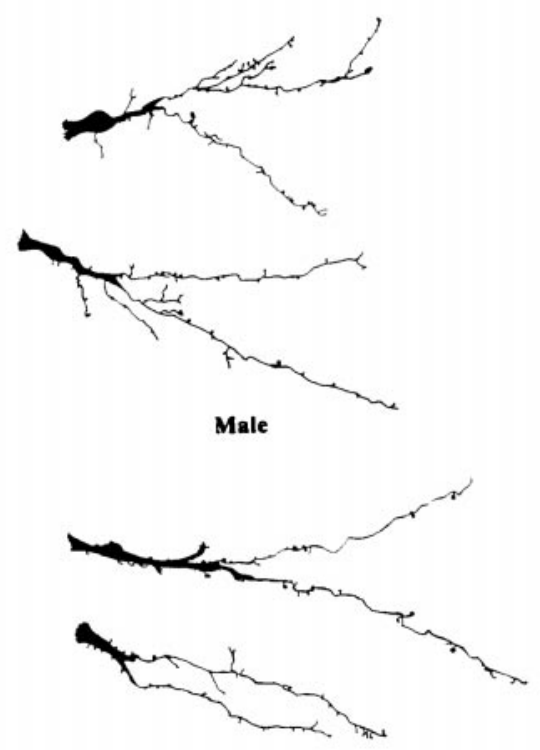

Castrate Male

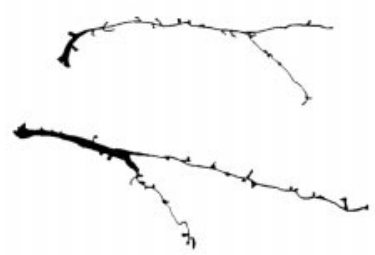

Female
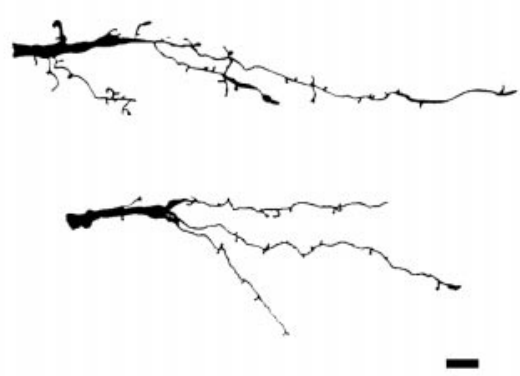

Female + TP
Figure 3. Camera lucida drawings of $A R C$ and $V M N$ dendrites from the hormonally treated groups. Drawings represent individual primary dendrites impregnated with Golgi-Cox from the $A R C(A)$ and $V M N(B)$ across four treatment groups. $A R C$ dendrites from the groups with high levels of circulating testosterone (males and females $+T P$ ) have significantly fewer spines on their dendrites than do groups with low levels of testosterone (females and castrate males). Dendrites in the $V M N$ have significantly more branch points in groups with high levels of circulating testosterone than do the groups with lower levels. Scale bars, $10 \mu \mathrm{m}$. three primary dendrites that branched occasionally. In contrast, the neurons in the VMN appeared much more complex with a more triangular-shaped soma and approximately four to five primary dendrites with several degrees of higher order branches.

The total length of dendrites in the VMN and ARC was not influenced by the hormonal status of the animal $\left[F_{(1,46)}=0.78\right.$ and $F_{(1,46)}=0.13$, respectively]. However, the mean dendritic length of neurons when pooled from the treatment groups was $19 \%$ longer in the VMN $(238.3 \pm 5.9 \mu \mathrm{m})$ than that in the ARC $\left[192.5 \pm 6.9 \mu \mathrm{m} ; F_{(1,53)}=24.96 ; p<0.0001\right]$. In contrast to the lack of a hormonal treatment effect on dendritic length, there was a significant effect on the number of branches on primary dendrites $\left[F_{(1,46)}=10.63 ; p<0.0001\right]$. Post hoc analysis revealed that only neurons in the VMN responded to testosterone with an increased branching; females and castrate males had 43 and $57 \%$ fewer branches, respectively, on their VMN dendrites than did males and females plus TP ( $p<0.01$; Figs. 3, 4A,C). There was no effect of hormonal status on dendritic branching in the ARC. However, the main effect of treatment on spine density was in the ARC $\left[F_{(1,46)}=7.62 ; p<0.001\right]$; females and castrate males had $37 \%$ more spines on their dendrites than did males and females plus TP ( $p<0.01$; Figs. 3, $4 B, D)$. There was no effect of treatment on the spine density in the $\operatorname{VMN}\left[F_{(1,46)}=1.10 ; p>0.05\right]$.

\section{Astrocytic morphology}

GFAP-IR was detectable in both the ARC and VMN. However, there were clear differences in the pattern of GFAP-IR between the two regions in that the staining in the VMN was more diff use and had the appearance of radial glia. Only a few differentiated astrocytes were observed in the VMN at PN2, and these were not readily amenable to quantification. These observations suggested that the radial glia had not yet differentiated into astrocytes and 
A

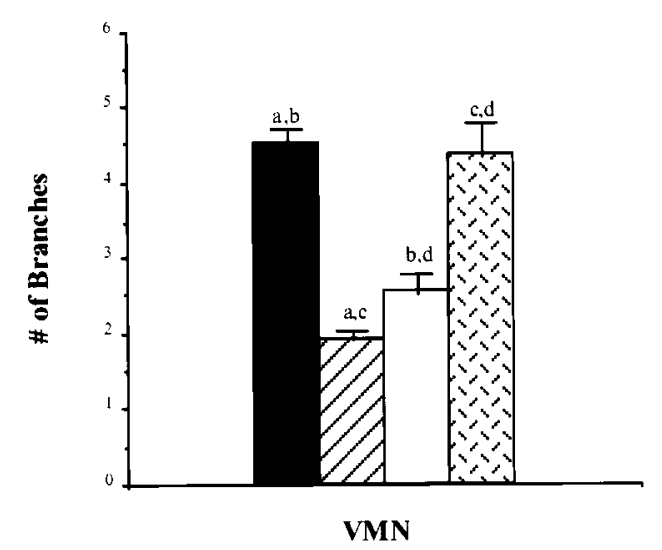

- INTACT MALE U CASTRATE MALE

$\mathrm{C}$

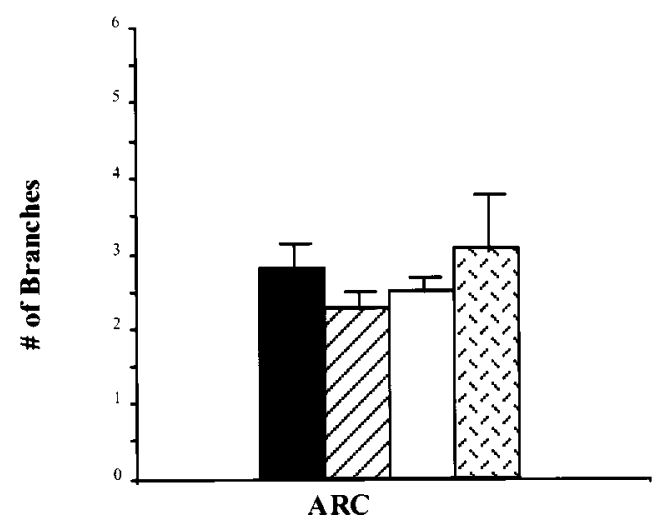

B

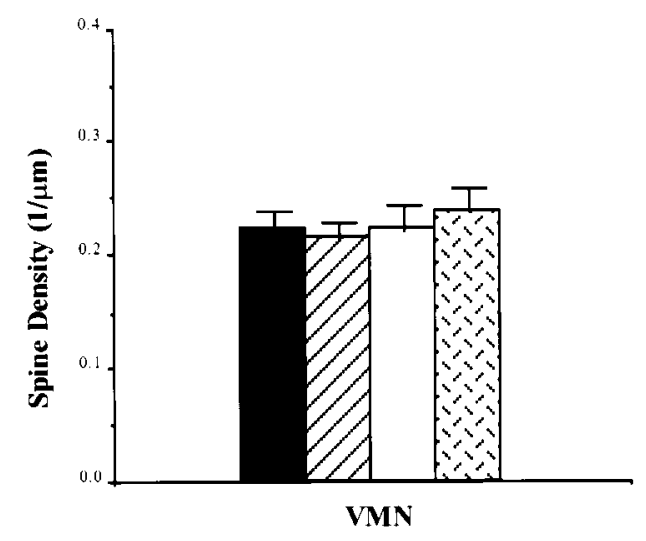

$\mathrm{D}$

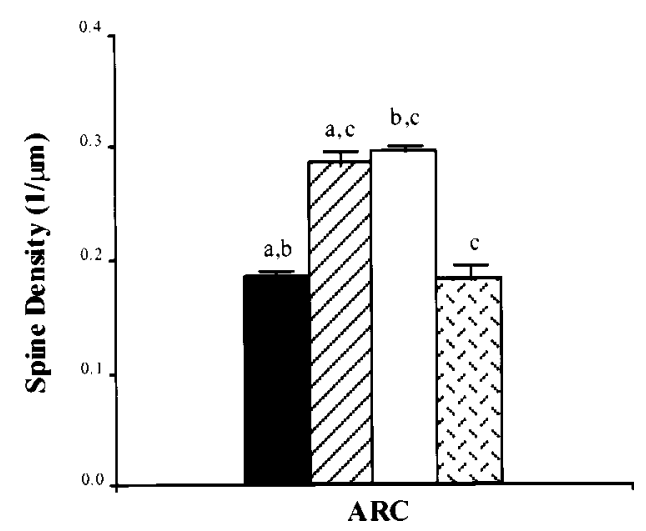

Figure 4. Effects of neonatal hormonal manipulation on neuronal morphology in the $A R C$ and $V M N$. Neuronal morphology was assessed using the Neurolucida system to reconstruct Golgi-Cox-impregnated neurons in the hypothalamus of PN2 rats. Approximately $10-20$ dendrites per animal in six to eight animals per group were analyzed. Data are the mean ( \pm SEM) of the number of branches on primary dendrites and the spine densities from the four treatment groups. $A, C$, The presence of gonadal steroids affected the number of branches on primary dendrites in the $V M N$ but not in the $A R C$. $B, D$, Conversely, the spine density of dendrites in the $A R C$ but not in the $V M N$ was affected by the gonadal steroids. Groups sharing the same letters $(a-d)$ in the individual graphs are significantly different from each other (ANOVA, $p<0.01$ ).

may express both vimentin, a marker for immature astrocytes, and GFAP as intermediate filament proteins (Schnitzer et al., 1981). In contrast, the GFAP-IR cells in the ARC have a range of appearance from fully stellate cells with bushy processes to simple bipolar cells with only a few processes. There were no radial glia-like cells immunoreactive for the GFAP antigen in the ARC at PN2.

In addition to the regional differences, the morphology of GFAP-IR astrocytes in the ARC was affected by hormonal manipulations, whereas the astrocytes in the VMN did not appear to be affected qualitatively. This is in part because there were too few differentiated cells to measure accurately in the VMN. In the ARC, GFAP-IR processes are $40 \%$ longer in gonadally intact males and females injected with TP than in females or castrate males $\left[F_{(1,16)}=46.75 ; p<0.01\right.$; Figs. 5, 6A]. The GFAP-IR astrocytes in the ARC were also categorized by class according to their degree of differentiation. In intact males and females injected with TP, $\sim 50 \%$ of the total GFAP-IR astrocytes were class IV or fully differentiated, and $\sim 5-10 \%$ were class I or undifferentiated astrocytes. In contrast, the ARC of castrate males and intact females was almost exactly the opposite, being composed of $50 \%$ class I and $<15 \%$ class IV GFAP-IR astrocytes $\left(\chi^{2}=162.4\right.$; $p<0.01$; Figs. 5, 6B).

\section{GFAP protein levels}

Using computerized densitometry, we detected a significantly lower level of GFAP immunoreactivity in the protein from the $\mathrm{VMN}$ of PN2 rats compared with that in the protein from the 

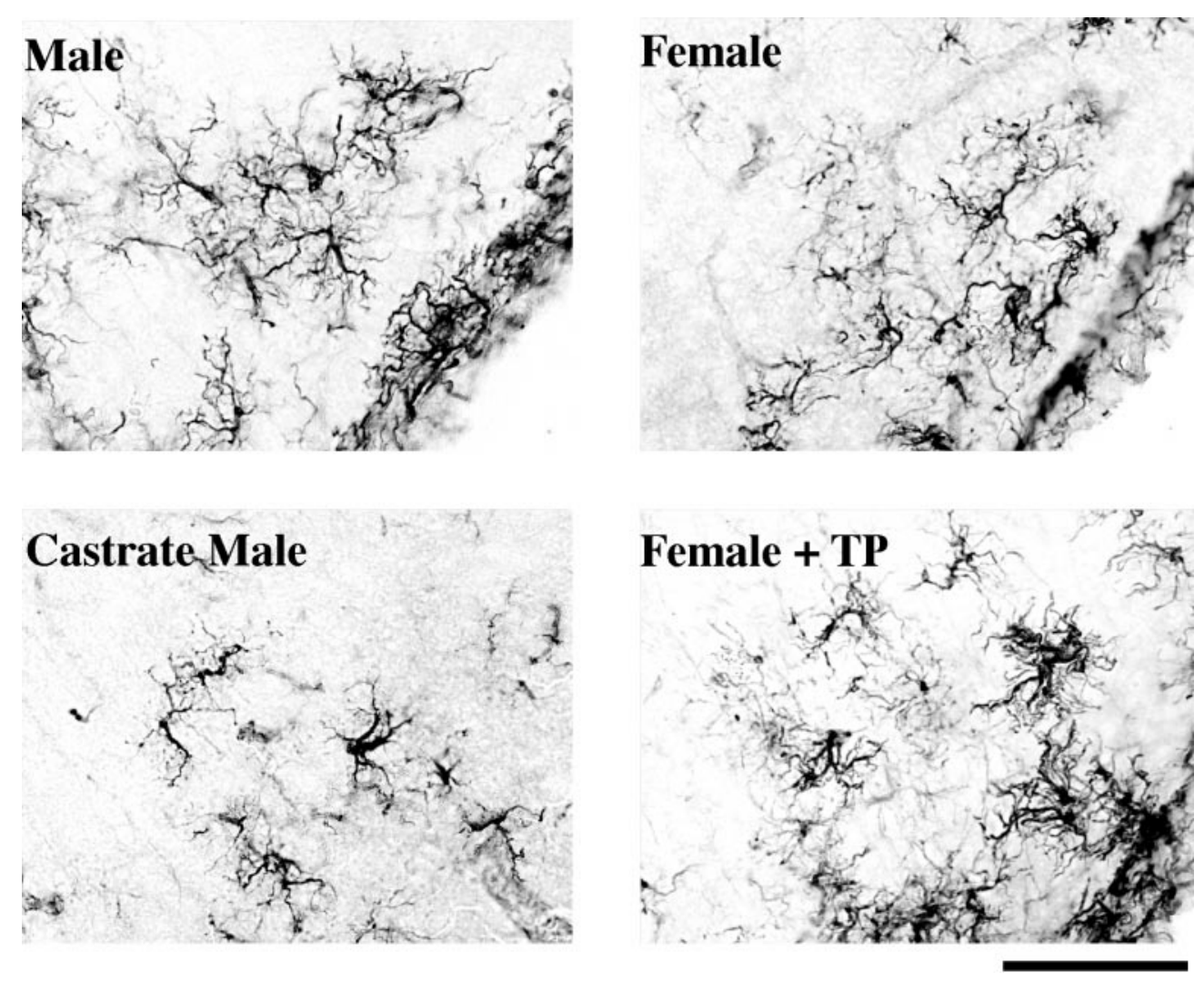

Figure 5. Representative photomicrographs $(20 \times)$ of ARC GFAP-IR astrocytes across different hormonal treatments. Males and females injected with $T P$ have a greater frequency of fully differentiated GFAP-IR astrocytes when compared with females and castrate males. Scale bar, $50 \mu \mathrm{m}$.

ARC of the same animals $(n=7)$. The ratio of the optical density of the GFAP- and GAPDH-immunoreactive bands from the ARC was $\sim 100 \times$ more dense than the optical density ratio of the bands from the VMN ( $t=3.05$; df $=12$; $p<0.02$; Fig. $7 A, B$.). The immunoreactive optical density of the housekeeping protein GAPDH was not significantly different between the ARC and VMN. GFAP and GAPDH were represented as immunoreactive bands at 51 and $36 \mathrm{kDa}$, respectively.

\section{DISCUSSION}

Analysis of neuronal and astrocytic morphology in the ARC and VMN during postnatal development has revealed a complex set of interactions that are both hormonally regulated and regionally specific. One of the most striking observations was that in the ARC of animals with high circulating levels of testosterone there was a coincident change in neuronal and astrocytic morphology in response to steroids. In both gonadally intact males and females injected with TP, there was an increase in astrocyte process length and the frequency of fully differentiated astrocytes. At the same time in animals with the same hormonal profile, there was a decrease in dendritic spine density. The reverse was true in the absence of high levels of testosterone; ARC dendrites had an increased dendritic spine density, and the astrocytes were less differentiated with shorter processes. In contrast, in the VMN there was no effect of TP on dendritic spine density. However, neurons in the VMN of males and of females injected with TP had a higher degree of branching when compared with oilinjected females and castrate males. Furthermore, there was no effect of TP on the morphology of astrocytes in the VMN in these animals. In fact, there appeared to be regional differences in astrocytic cell morphology between the ARC and VMN in that differentiated astrocytes were virtually nonexistent at this age in the VMN regardless of hormonal status, a conclusion supported by the observation of extremely low levels of GFAP in the VMN compared with the ARC. Unrelated to the hormonal effects, developing neurons in the VMN had longer dendrites than neurons in the ARC.

To our knowledge this is the first demonstration of coordinated morphological plasticity in neurons and astrocytes in developing rat brain in vivo. Several in vivo studies of adult rat hypothalamic nuclei demonstrate neuronal plasticity in response to alterations of the hormonal milieu in which the neural substrates in both male and female adult rat ARC and VMN undergo dramatic structural modifications (Olmos et al., 1989; Frankfurt et al., 1990; Segarra and McEwen, 1991; Danzer et al., 1998). A major component of neuronal plasticity appears to be changes in dendritic spine density, and estrogen has been found to influence the dendritic spine density in adult hippocampus and VMN (Frankfurt et al., 1990; Gould et al., 1990; Frankfurt and McEwen, 1991; Segarra and McEwen, 1991; Woolley and McEwen, 1992). Dendritic spines are the site of excitatory synapses, and analyses at the electron microscopy level demonstrate that hormonally induced spines result in increased numbers of synapses (Woolley and McEwen, 1992; Woolley et al., 1996). Thus, sexually dimorphic dendritic spine densities in the ARC of $\mathrm{PN} 2$ rats may provide the synaptic framework for sexually dimorphic neuroendocrine secretions and behaviors exhibited by adult rats.

\section{Neuronal-glial interactions}

Neuronal-glial interactions within the CNS begin during early stages of fetal development and extend throughout the lifespan of adult rats, with glia playing different roles at different stages. During neurogenesis and early development, radial glia provide a scaffold for migrating neurons (Rakic, 1972). These radial glia serve as precursors to the more mature astrocytes. In vitro studies suggest that astrocytes play an active role in determining region- 
A

Figure 6. Analysis of $A R C$ GFAP-IR astrocytes. $A$, Effects of neonatal hormonal manipulation on GFAP-IR process length. The mean distance that processes extended from the astrocyte soma was measured in $28-40$ cells per animal and four animals per group. Increased process length is correlated with increased differentiation. Data are the mean ( \pm SEM) of GFAP-IR process length in the $A R C$ of four treatment groups in PN2 rats. Groups sharing common letters $(a-d)$ are significantly different from each other (ANOVA, $p<0.01$ ). The process length was significantly longer in intact males and in females injected with TP compared with castrate males and intact females. $B$, The morphological appearance of astrocytes in the PN2 ARC. Astrocytes were categorized into four classes (see Materials and Methods; Fig. 5 ), and the percentage of each in the four treatment groups was quantified. There was a significant difference in the distribution of cell classes with intact males and females injected with TP having a higher frequency of class IV cells that represent the greatest degree of differentiation. Conversely, castrate males and intact females have more class I cells that lack a high degree of differentiation $\left(\chi^{2}, p<0.01\right)$.

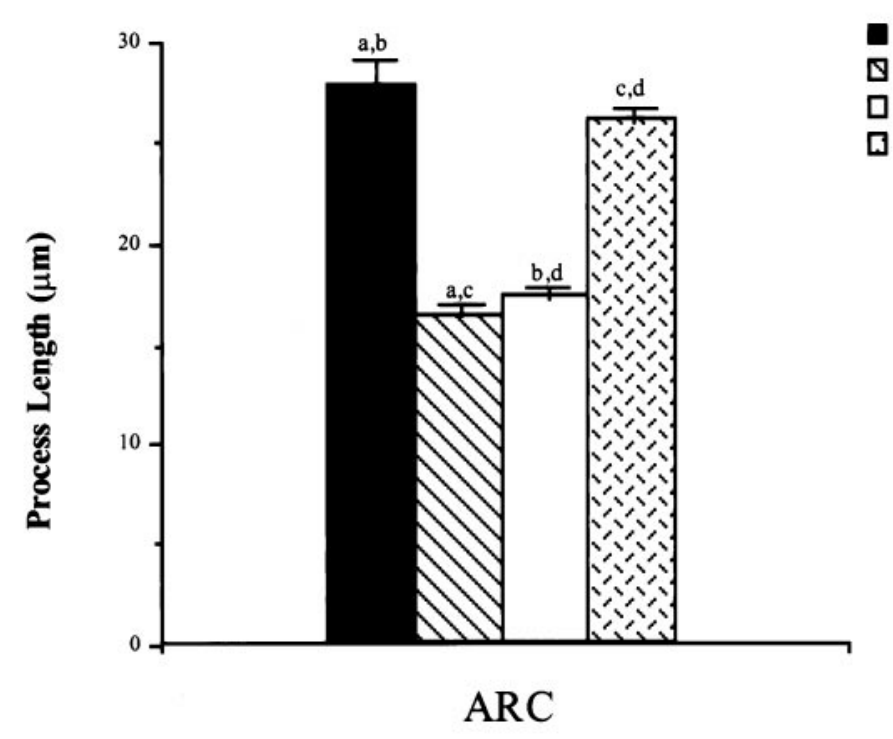

B

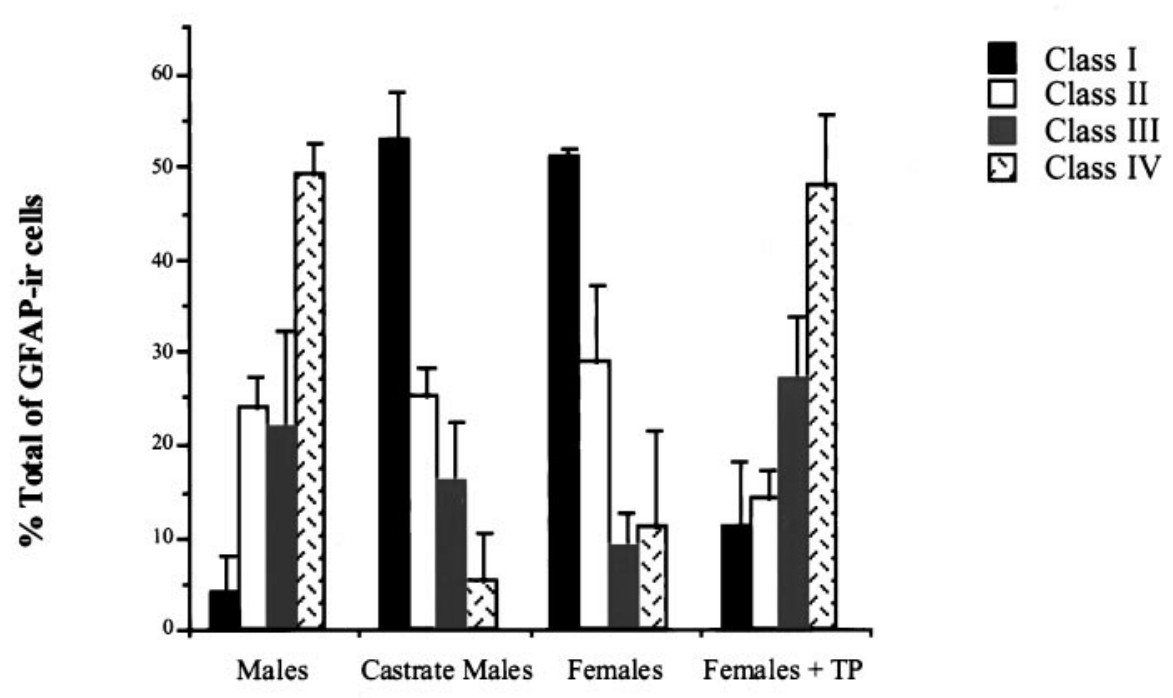

ARC specific patterns of synapse formation in developing brain, as well as participating in synaptic plasticity in adult CNS. For example, Le Roux and Reh (1995) have demonstrated that embryonic cortical neurons developed three times more primary dendritic outgrowths when cultured with astrocytes derived from the cortex, retina, and olfactory bulb than on glia derived from the mesencephalon, striatum, or spinal cord of postnatal rats. Moreover, they suggest that this effect is mediated by a diff usible factor because the glia-conditioned media elicited the same results. Alternatively, nondiffusible factors from astrocytes may also mediate changes in neuronal morphology. In contrast to radial glia, mature astrocytes synthesize extracellular matrix molecules such as tenascin and chondroitin sulfate proteoglycans that are known to be nonpermissive to neurite outgrowth (McKeon et al., 1991; Faissner and Steindler, 1995).

A hallmark of astrocyte maturation is increased synthesis of
GFAP (Eng, 1985; Condorelli et al., 1990; Eng and Ghirnikar, 1994). The intermediate filaments of astrocytes are composed of the CNS-specific GFAP and the less cell-specific filament protein vimentin (Bignami et al., 1972). Vimentin is the major cytoskeletal protein expressed in early astrocytic development (Dahl et al., 1981; Schnitzer et al., 1981). As development progresses, radial glia are believed to be transformed into stellate process-bearing astrocytes (Schmechel and Rakic, 1979; Ramon y Cajal, 1995) that are marked by an accumulation of GFAP (Culican et al., 1990), and stellation of astrocytes is generally considered a characteristic of fully differentiated astrocytes both in vivo and in vitro (Hatten, 1983; Grasser and Hatten, 1990). Our analysis of regional GFAP expression by Western blots, in combination with our visual observations, suggests that ARC astrocytes exhibit a greater degree of maturation than do astrocytes in the VMN, which may be the consequence of an intrinsic developmental program. Thus, the lack 
A

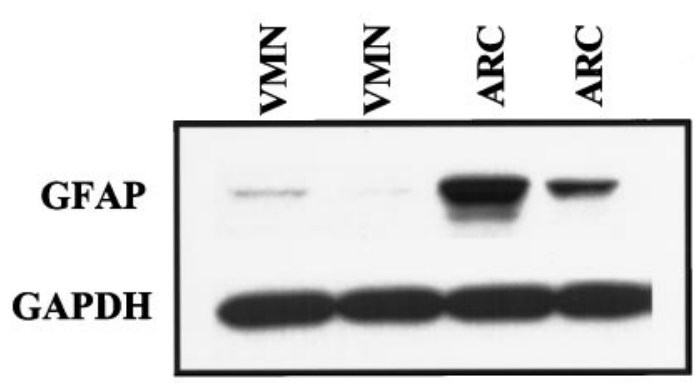

B

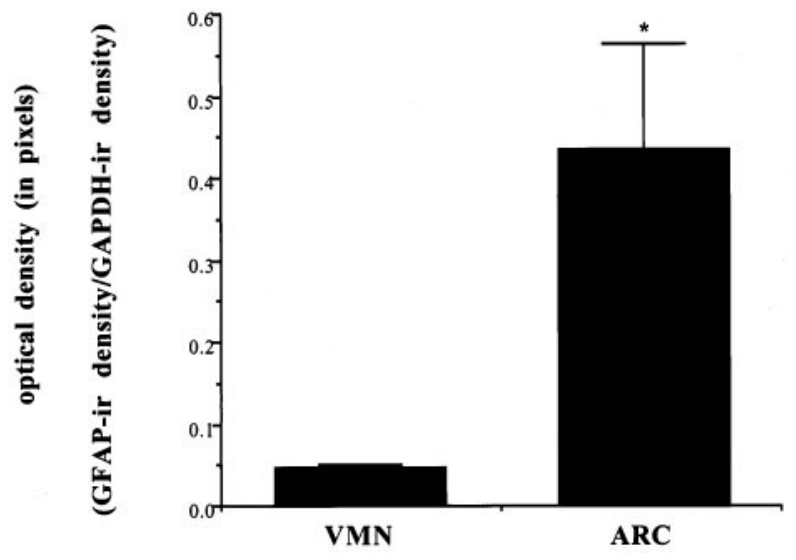

Figure 7. Western blot analysis. $A$, Four representative electrophoretic lanes from an immunoblot of tissue micropunches from the VMN (lanes 1, 2 ) and the $A R C$ (lanes 3, 4) of two animals. Lanes 1 and 3 and lanes 2 and 4 contain tissue from the same animal. A total of $5 \mu \mathrm{g}$ of total protein was loaded onto each lane. The blot was probed with a mouse monoclonal antibody to GFAP that recognized an appropriate band at $51 \mathrm{kDa}$ and with a mouse monoclonal to $G A P D H$ that recognized the appropriate 36 $\mathrm{kDa}$ band. $B$, Levels of GFAP in the $V M N$ and $A R C$. Data are represented as a ratio of the optical density of the GFAP-immunoreactive band to that of the $G A P D H$-immunoreactive band. Levels were significantly higher in the $A R C$ compared with the $V M N$ of PN2 rats ( $p<0.02, t$ test).

of steroid-induced neuronal branching in the ARC may be attributable to the presence of mature and/or maturing astrocytes resulting in a nonpermissive environment for dendritic growth.

\section{Steroid-induced neuronal-glial interactions}

In the adult rat hypothalamus, astrocyte morphology changes in response to a specific stimulus such as steroids, and the resulting morphology influences neighboring synapses (Theodosis and Poulain, 1993; Garcia-Segura et al., 1994b). Work by Garcia-Segura and colleagues demonstrates the role of neuronal-astrocytic interactions on synaptic remodeling of adult female rat ARC over the estrous cycle. When estrogen levels are high on the day of proestrus, glia in the ARC increase their surface area (GarciaSegura et al., 1994a). Electron microscopy studies suggest that extended astrocytic processes decrease inhibitory synaptic inputs by ensheathing ARC neurons (Garcia-Segura et al., 1994b). This "phasic synaptic remodeling" of the ARC in response to estrogen is sexually dimorphic. Brains masculinized by gonadal steroid exposure during the critical developmental period of brain sex differentiation no longer exhibit this plasticity (Garcia-Segura et al., 1994b). The current data suggest that early differentiation of ARC astrocytes after exposure to testosterone and its metabolite estrogen may be causally related to the loss of plasticity in the adult male.

The majority of sex differences in the rodent brain are caused by local conversion of testosterone to estrogen by the p450 aromatase enzyme (Naftolin et al., 1975; McEwen et al., 1977; Lephart, 1996) that is localized in neurons and does not appear to be present in astrocytes (Martini and Melcangi, 1991). We have confirmed that neonatal ARC glia are also responding to locally synthesized estrogen, rather than to the testosterone itself (Mong and McCarthy, 1998). However, whether estrogen is acting directly on neurons, astrocytes, or both is unknown. Steroids, such as estrogen, principally act via intracellular receptors that translocate to the nucleus after binding and alter gene transcription. Estrogen receptor protein and mRNA have been reported in cultured astrocytes from several brain regions (Jung-Testas et al., 1992; Santagati et al., 1994; Murphy et al., 1998) including the hypothalamus (Ma et al., 1994; Ma and Ojeda, 1997), and estrogen receptors have been immunocytochemically detected in guinea pig glia in the preoptic area and the median eminence (Langub and Watson, 1992). However, there has been no clear in vivo demonstration of any gonadal steroid receptors in hypothalamic astrocytes of neonatal rats (Garcia-Segura et al., 1996). Moreover, neonatal rat hypothalamic astrocytes in vitro differentiate in the presence of estrogen only when cocultured with hypothalamic neurons (Torres-Aleman et al., 1992), and the presence of neurons in astrocytic cultures has been shown to reverse the effect of estrogen on GFAP mRNA synthesis (Stone et al., 1998). Therefore, the cellular site of estrogen action remains unclear. In view of this, we envision three possible scenarios for estrogen-induced changes in neuronal-astrocytic morphology in the neonate. First, in the ARC, neurons convert testosterone into estrogen where it initiates a signaling cascade that transduces this signal to the surrounding astrocytes to increase differentiation. A signal from the differentiated astrocytes in turn attenuates neuronal formation of dendritic spines. The decrease in spine formation in the ARC may be the result of a physical blockade by astrocytic processes or by some diffusible factor that is released from glia and signals back to the neuron. In contrast, either astrocytes in the VMN are not sufficiently mature to respond to a differentiating signal from the neurons, or neurons of the VMN do not respond to estrogen with the production of such a signal. Regardless, the lack of astrocyte differentiation in the VMN may create a permissive environment for estrogen-induced dendritic growth and branching. The second possibility is that estrogen acts directly on neurons in both the ARC and VMN to influence changes in neuronal morphology in a regionally specific manner and mediates the release of an astrocytic differentiating factor in the ARC. Third, it is possible that estrogen is acting directly on ARC astrocytes to induce their differentiation either via steroid receptors or via other nontraditional pathways and that changes in neuronal and astrocytic morphology are independent of each other. Our future experiments will distinguish between these scenarios.

\section{REFERENCES}

Altman J, Bayer SA (1995) Atlas of prenatal rat brain development. Ann Arbor, MI: CRC.

Arnold AP, Gorski RA (1984) Gonadal steroid induction of structural sex differences in the central nervous system. Annu Rev Neurosci 7:413-442.

Bignami A, Eng LF, Dahl D, Uyeda CT (1972) Localization of glial fibrillary acidic protein in astrocytes by immunofluorescence. Brain Res 430:113-126.

Condorelli DF, Dell'Albani P, Kaczmarek L, Messina L, Spamponato G, 
Avola R, Messina A, Giuffrida Stella AM (1990) Glial fibrillary acidic protein messenger mRNA and glutamine synthetase activity after nervous system injury. J Neurosci Res 26:251-257.

Culican SM, Baumrind NL, Yamamoto M, Pearlman AL (1990) Cortical radial glia: identification in tissue culture and evidence for transformation to astrocytes. J Neurosci 10:684-692.

Dahl D, Rueger DC, Bignami A (1981) Vimentin, the 57,000 M.W. protein of fibroblast filament, is the major cytoskeletal component of immature glia. Eur J Cell Biol 24:191-196.

Danzer SC, McMullen NT, Rance NE (1998) Dendritic growth of arcuate neuroendocrine neurons following orchidectomy in adult rats. J Comp Neurol 390:234-246.

Eng LF (1985) GFAP: the major protein of glial intermediate filaments in differentiated astrocytes. J Neuroimmunol 8:203-214.

Eng LF, Ghirnikar RS (1994) GFAP and astrogliosis. Brain Pathol 4:229-237.

Faissner A, Steindler D (1995) Boundaries and inhibitory molecules in developing tissues. Glia 13:233-254.

Forgie ML, Gibb R, Kolb B (1996) Unilateral lesions of the forelimb area of rat motor cortex: lack of evidence for use-dependent neural growth in the undamaged hemisphere. Brain Res 710:249-259.

Frankfurt M, McEwen BS (1991) 5,7-Dihydroxytryptamine and gonadal steroid manipulation alter spine density in the ventromedial hypothalamic neurons. Neuroendocrinology 54:653-657.

Frankfurt M, Gould E, Woolley CS, McEwen BS (1990) Gonadal steroids modify dendritic spine density in the ventromedial hypothalamic neurons: a Golgi study in the adult rat. Neuroendocrinology 51:530-535.

Garcia-Segura LM, Luquin S, Parducz A, Naftolin F (1994a) Gonadal hormone regulation of glial fibrillary acidic protein immunoreactivity and glial ultrastructure in the rat neuroendocrine hypothalamus. Glia 10:59-69.

Garcia-Segura LM, Chowen JA, Duenanas M, Torres-Aleman I, Naftolin F (1994b) Gonadal steroids as promoters of neuro-glial plasticity. Psychoneuroendocrinology 19:317-345.

Garcia-Segura LM, Chowen JA, Naftolin F (1996) Endocrine glia: roles of glial cells in the brain actions of steroids and thyroid hormones and in the regulation of hormone secretion. Front Neuroendocrinol 17:180-211.

Gerall AA, Moltz H, Ward IL (1992) Handbook of behavioral neurobiology: sexual differentiation. New York: Plenum.

Gibb R, Kolb B (1998) A method for vibratome sectioning of Golgi-Cox stained whole rat brain. J Neurosci Methods 79:1-4.

Glaser EM, Van der Loos H (1981) Analysis of thick sections by obversereverse computer microscopy: application of a new, high clarity GolgiNissl stain. J Neurosci Methods 4:117-125.

Gould E, Woolley CS, Frankfurt M, McEwen BS (1990) Gonadal steroids regulate dendritic spine density in hippocampal pyramidal cells in adulthood. Neuroscience 10:1286-1291.

Grasser UE, Hatten ME (1990) Neuron-glia interactions of rat hippocampal cells in vitro. Glial-guided neuronal migration and neuronal regulation of glial differentiation. J Neurosci 10:1278-1285.

Hatten ME (1983) Embryonic cerebellar astroglia in vitro. Dev Brain Res 13:309-313.

Jung-Testas I, Renoir M, Bugnard H, Greene GL, Baulieu EE (1992) Demonstration of steroid hormone receptors and steroid action in primary cultures of rat glial cells. J Steroid Biochem Mol Biol 41:621-631.

Laemmli UK (1970) Cleavage of structural proteins during the assembly of the head of bacteriophage $\mathrm{T}_{4}$. Nature 227:680-685.

Langub MC, Watson RE (1992) Estrogen receptor immunoreactive glia, endothelia, and ependyma in guinea pig preoptic area and median eminence: electron microscopy. Endocrinology 130:364-372.

Le Roux PD, Reh TA (1995) Independent regulation of primary dendritic and axonal growth by maturing astrocytes in vitro. Neurosci Lett 198:5-8.

Lephart ED (1996) A review of brain aromatase cytochrome P450. Brain Res Rev 22:1-26.

Ma YJ, Ojeda SR (1997) Neuroendocrine control of female puberty: glial and neuronal interactions. J Invest Dermatol Symp Proc 2:19-22.

Ma YJ, Berg-von der Emde K, Moholt-Siebert M, Hill DF, Ojeda SR (1994) Region specific regulation of transforming growth factor $\alpha$ $(\mathrm{TGF} \alpha)$ gene expression in astrocytes of the neuroendocrine brain. J Neurosci 14:5644-5651.

Maclusky NJ, Naftolin F (1981) Sexual differentiation of the central nervous system. Science 211:1294-1303.

Martini L, Melcangi RC (1991) Androgen metabolism in the brain. J Steroid Biochem Mol Biol 47:195-205.
Matsumoto A, Arai Y (1980) Sex dimorphism in "wiring pattern" in the hypothalamic arcuate nucleus and its modification by neonatal hormonal environment. Brain Res 190:238-242.

Matsumoto A, Arai Y (1986) Male-female differences in synaptic organization of the ventromedial nucleus of the hypothalamus in rats. Neuroendocrinology 42:232-236.

McEwen BS, Lieberburg I, Chaptal C, Krey LC (1977) Aromatization: important for sexual differentiation of the neonatal rat brain. Horm Behav 9:249-263.

McKeon RJ, Schreiber RC, Rudge JS, Silver J (1991) Reduction of neurite outgrowth in a model of glial scarring following CNS injury is correlated with the expression of inhibitory molecules on reactive astrocytes. J Neurosci 11:3398-3411.

Mong JA, McCarthy MM (1998) Estrogen mediates the hormonal responsiveness of arcuate astrocytes in neonatal rats. Soc Neurosci Abstr 24:220.

Mong JA, Kurzweil RL, Davis AM, Rocca MS, McCarthy MM (1996) Evidence for sexual differentiation of glia in rat brain. Horm Behav 30:553-562.

Murphy DD, Cole NB, Greeneberger V, Segal M (1998) Estradiol increases dendritic spine density by reducing GABA neurotransmission in hippocampal neurons. J Neurosci 18:2550-2559.

Naftolin F, Ryan KJ, Davies IJ, Petro Z, White RJ, Wolin L (1975) The formation of estrogen by central neuroendocrine tissue. Recent Prog Horm Res 31:295-319.

Ojeda SR, Urbanski HF (1994) Puberty in the rat. In: Physiology of reproduction (Knobil E, Neill JD, eds), pp 453-486. New York: Raven.

Olmos G, Naftolin F, Peres J, Tranque PA, Garcia-Segura LM (1989) Synaptic remodeling in the rat arcuate nucleus during the estrous cycle. Neuroscience 32:663-667.

Palkovits M, Brownstein MJ (1988) Maps and guide to microdissection of the rat brain. New York: Elsevier.

Pfaff DW, Schwartz-Giblin S, McCarthy MM, Kow L-M (1994) Cellular and molecular mechanisms of female reproductive behaviors. In: Physiology of reproduction (Knobil E, Neill JD, eds), pp 107-220. New York: Raven.

Rakic P (1972) Mode of cell migration to the superficial layers of fetal monkey neocortex. J Comp Neurol 145:61-84.

Ramon y Cajal S (1995) Histology of the nervous system. New York: Oxford UP.

Santagati S, Melcangi RC, Celtotti F, Martini L, Maggi A (1994) Estrogen receptor is expressed in different types of glial cells in culture. J Neurochem 63:2058-2064.

Schmechel DE, Rakic P (1979) A Golgi study of radial glial cells in developing monkey telencephalon: morphogenesis and transformation into astrocytes. Anat Embryol (Berl) 156:115-152.

Schnitzer J, Franke WW, Schachner M (1981) Immunocytochemical demonstration of vimentin in astrocytes and ependymal cells of developing and adult mouse nervous system. J Cell Biol 90:435-447.

Segarra A, McEwen BS (1991) Estrogen increases spine density in the ventromedial hypothalamic neurons of peripubertal rats. Neuroendocrinology 54:365-372.

Stone DJ, Song Y, Anderson CP, Krohn KK, Finch CE, Rozovsky I (1998) Bidirectional transcription regulation of glial fibrillary acidic protein by estradiol in vivo and in vitro. Endocrinology 139:3202-3209.

Theodosis D, Poulain D (1993) Activity-dependent neuronal-glial and synaptic plasticity in the adult mammalian hypothalamus. Neuroscience 57:501-535.

Torres-Aleman I, Rejas MT, Pons S, Garcia-Segura LM (1992) Estradiol promotes cell shape changes and fibrillary acidic protein redistribution in hypothalamic astrocytes in vitro: a neuronal mediated effect. Glia 6:108-117.

Towbin H, Staehlin T, Gordon J (1979) Electrophoretic transfer of proteins from polyacrylamide gels to nitrocellulose sheets: procedure and some applications. Proc Natl Acad Sci USA 76:4350-4354.

Weisz J, Ward IL (1980) Plasma testosterone and progesterone titers of pregnant rats, their male and female fetuses and neonatal offspring. Endocrinology 106:306-313.

Woolley C, Wensel HJ, Schwartzkroin PA (1996) Estradiol increases the frequency of multiple synapse boutons in the hippocampal CA1 region of the adult female rat. J Comp Neurol 373:108-117.

Woolley CS, McEwen BS (1992) Estradiol mediates fluctuation in hippocampal synapse density during the estrous cycle in the adult rat. J Neurosci 12:2549-2554. 